\title{
¿Es importante la comida en China? Cuestiones de política agrícola moderna
}

Does Food Matter in China? Issues of Modern Agricultural Policy

Yolanda Trápaga Delfin*

\section{Resumen}

El éxito económico de la República Popular China a partir de las reformas económicas de 1978 tiene como uno de sus pilares al sector agropecuario. La planificación central de la producción, con el objetivo de autosuficiencia en trigo, maíz y arroz, es el eje de la política sectorial que se considera como un renglón estratégico. Sin embargo, este modelo enfrenta importantes restricciones, tales como la alta ineficiencia del patrón alimentario adoptado, la escasez relativa de suelos y agua, la contaminación de que han sido objeto, la desertificación del territorio, los cambios de uso del suelo a favor de la industrialización y la urbanización. Para lidiar con estos problemas, el gobierno chino realiza importantes inversiones, principalmente en países de África y América Latina, con el fin de ampliar su frontera agrícola y mantener el objetivo de la autosuficiencia. Nos preguntamos si esta política puede seguir en el largo plazo.

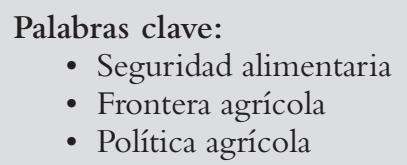

Hablar de la capacidad de China para producir alimentos es un tema muy relevante, tanto dentro de sus fronteras como para el mundo. En términos económicos sabemos que los alimentos son un componente primordial de la canasta básica de los trabajadores, sobre todo cuando los ingresos son bajos. Además, la producción de alimentos tiene el poder de estructurar el patrón de cultivos vigente en un país que pretende la autosuficiencia en ese rubro. Para China, frecuentemente se parte de subrayar que debe alimentar a la porción de población más numerosa del planeta con recursos naturales muy escasos para la producción agropecuaria, por lo que resulta útil entender el alcance y los límites de la política agroalimentaria con la que su gobierno ha logrado sustentar una exitosa acumulación originaria de última generación, basada

* Profesora titular adscrita a la División de Estudios de Posgrado de la Facultad de Economía de la UnAm. Responsable del Centro de Estudios China México. - " * 
simultáneamente en la relación capital-trabajo asalariado y en la existencia de esquemas económicos no convencionales para el capital que son usados para el buen funcionamiento del modelo "con características chinas", tales como formas de propiedad de todo tipo, producción centralmente planificada, control territorial y demográfico de la fuerza de trabajo, así como ignorar la teoría de las ventajas comparativas en aras de proteger sectores considerados por el Partido Comunista Chino (PCC) como "vitales para la economía nacional" (OMC, 2012 b). Tal es el caso de la producción de granos.

Con sólo $8 \%$ de la tierra agrícola del mundo y $6 \%$ de las fuentes renovables de agua dulce, China es la nación con el sector agrícola más grande del mundo y uno de los principales importadores y exportadores de productos del campo. Su importancia para el mundo es indiscutible, en 2013 era ya el mayor productor de arroz, trigo, puercos, huevos, algodón, frutas y hortalizas, así como el segundo en maíz y carne de aves. Es apenas en 2011 que China deja de ser un país rural, pues la mayor parte de sus habitantes vive ya en asentamientos urbanos, pero la pregunta de ¿quién alimentará a su población? tiene tanta importancia como la ha tenido en su larga historia. Se la han hecho dentro y fuera del país funcionarios, especialistas, las empresas transnacionales, los gobiernos de las economías exportadoras netas de alimentos, así como los países importadores que ven en el incremento de la demanda china un foco rojo por su posible impacto en los precios o por su capacidad para generar escasez en un momento dado. Pero la respuesta que ha guiado la historia de la República Popular es: "Nosotros, los chinos, nos alimentaremos a nosotros mismos" (Chinese Government, 2006a).

Con el fin de obtener posibles lecciones útiles para los países deficitarios o con restricciones de recursos para la producción del campo, en estas páginas haremos un breve examen del contexto en que se producen los alimentos para un quinto de la población mundial, prácticamente cautiva dentro de sus fronteras y con ingresos al alza, así como para sostener el crecimiento de la economía emergente más dinámica y la segunda en el mundo por su tamaño. Hay que saber entonces qué condiciona la política agrícola del Imperio Medio y qué tendencias están presentes para entender su inserción en los mercados globales de alimentos.

\section{Antecedentes}

Desde la fundación de la República Popular China en 1949, el gobierno de Mao Tse Dong tuvo como uno de sus objetivos más importantes garantizar 
con fuentes domésticas el abasto de una canasta mínima de alimentos para la población. Sin embargo, con base en el modelo de colectivización de la tierra de inspiración soviética y de las comunas populares no se obtuvo el éxito esperado pues no se logró resolver satisfactoriamente la generación estable y suficiente de alimentos, mucho menos una canasta variada; de tal manera que entre 1958 y 1961, durante el periodo del Gran Salto Adelante, China vive una devastadora hambruna en la que murieron cuando menos 30 millones de personas. Desde entonces y hasta la muerte de Mao en 1976 fueron normales el racionamiento y la generalización de una dieta muy elemental basada en granos, tubérculos, pocas hortalizas y esporádicamente productos de la ganadería.

Formalmente es a partir de 1978, al cambiar la política económica hacia una de acumulación en zonas especiales basada en la relación capital-fuerza de trabajo, que el primer sector a ser reformado de manera radical es el rural, para de ahí estructurar la apertura de la economía, la inserción del país en la globalización y el modelo de acumulación originaria con estilo chino del que el mundo ha sido testigo, pero donde el campo no recibió los beneficios del proceso, pues igual que en el pasado fueron primordialmente para el sector urbano.

\section{La estructura para la producción de alimentos}

En ausencia de capital, las reformas económicas fuera de la agricultura se anclan en la ventaja comparativa de la abundancia de fuerza de trabajo y del establecimiento de ventajas extraordinarias para la inversión extranjera directa en zonas especiales de industrialización, mientras que en el campo se mantiene inamovible hasta hoy el eje de la producción de alimentos básicos en niveles de la mayor autosuficiencia posible; no se instalan las ventajas comparativas ni criterios de competitividad internacional, contribuyendo de esa manera en el proceso de acumulación en curso. ¿En qué radican entonces los cambios que decidió el Comité Central del PCC para el campo? ¿Cuál ha sido su aporte al éxito económico del país?

En primer lugar, se lleva a cabo una reforma agraria que pone fin a la colectivización del campo y, sin privatizarla, da la tierra en usufructo ${ }^{1}$ a cada ho-

${ }^{1}$ En China la propiedad del suelo es social y en el campo se da en usufructo a las familias en un sistema dinámico de redistribución permanente para recientemente permitir a los agricultores dar los derechos en alquiler, generando un mercado de esos derechos, pero asegurando que la propiedad permanezca en manos de la colectividad (Chinese Government, 2005). Los colectivos de las aldeas siguen siendo propietarios del suelo y emiten los contratos de uso del suelo por cuando menos 30 
gar campesino junto con la autorización de producir para el mercado después de cumplir con las cuotas obligatorias establecidas por el gobierno. El nuevo esquema se denomina sistema de responsabilidad familiar y es el que sigue vigente, asegurando que la propiedad permanezca en manos de la colectividad.

En segundo lugar, se cancela el monopolio estatal de la distribución del producto agrícola y el control de precios sobre los bienes no estratégicos.

En tercer lugar, se promueve el aumento de los ingresos de los 800 millones de agricultores existentes en esa época estimulando las empresas rurales ${ }^{2}$ ya existentes, así como la creación de nuevas, al mismo tiempo que se les permite establecer diversos tipos de negocios, rubro no autorizado hasta entonces (Chinese Government, 2006b). Estos elementos se combinan con lineamientos de política encaminados a aumentar la productividad agrícola por la vía del desarrollo de la ciencia y la tecnología en el renglón de la agronomía y creando una rama de biotecnología propia.

El modelo de las empresas rurales es fundamental para entender el arraigo de los agricultores en el campo, pues éstas permiten complementar los magros ingresos generados por su actividad primaria. Simultáneamente existe un elemento de primer orden y de validez universal para los ciudadanos chinos que es el "registro en el lugar de nacimiento" (hukou), o en el de la madre, que impide legalmente la libre movilidad de la población en el territorio chino. Es el complemento que logra impedir que con ingresos muy bajos en términos absolutos y en relación a los urbanos, se diera una expulsión masiva de campesinos hacia las ciudades en busca de mejores condiciones de vida; el hukou arraiga al ciudadano a su lugar de nacimiento, reconociendo la vigencia total de sus derechos sólo ahí, pues al impedir la libre circulación cancela sus posibilidades de obtener satisfactores básicos fuera de su demarcación - habitación, educación y atención de salud-, por convertirse prácticamente en ilegal dentro de su país. ${ }^{3}$ A pesar de ello, a fines de 2011 alrededor de 250 millones

años. Dependiendo del tipo de explotación (agrícola, forestal, praderas), el contrato puede ser hasta de 70 años. En el periodo de vigencia del contrato los agricultores individuales tienen garantizados sus derechos legales de ocupación, uso y percepción de las ganancias generadas por la explotación del suelo, pero no pueden vender la tierra, ni usarla como garantía.

${ }^{2}$ Las empresas rurales fueron creadas en la época del Gran Salto Adelante, entre 1958 y 1961, y un millón y medio fueron recuperadas con las reformas hasta alcanzar 12 millones en 1985. Participan en muchos sectores: manufacturas, procesamiento de productos agrícolas, transporte y comunicaciones, construcción, comercio y alimentación. En 2004, 22.13 millones de empresas rurales empleaban a 138.66 millones de habitantes del campo y en 2008 daban ocupación a 30\% de la población económicamente activa rural, con un valor agregado siempre en aumento (Chinese Government, 2006b).

${ }^{3}$ En el marco de su política de urbanización, en febrero de 2012 el Consejo de Estado dijo que 
de personas se habían trasladado a las áreas urbanas en busca de trabajo sin la residencia legal, según datos de la Agencia Central de Inteligencia de Estados Unidos (CIA, 2013).

La nueva estructura arrojó un rápido incremento del producto agrícola, en particular obteniendo la autosuficiencia en ciertos granos y en 2005 el país deja de ser beneficiario de ayuda alimentaria para convertirse en donante. Este marco de funcionamiento de la agricultura en China ha garantizado el abasto de granos a niveles cercanos a $100 \%$ por más de 30 años. Los objetivos del Plan Nacional de Largo Plazo para la Seguridad en Alimentos (2008-2020) consisten en estabilizar la cantidad de tierra cultivada, mantener la tasa de autosuficiencia en 95\%, y conservar un nivel razonable de reservas que en 2010 se estimaban en más de la mitad de $17 \%$ recomendado por FAO. Medido en valor, hoy China es el primer productor mundial de arroz, trigo, maíz, mijo, cebada, papas, cacahuates, té, manzanas, algodón, oleaginosas, puercos y pescado (CIA, 2013). Al mismo tiempo, la dieta de la población se ha diversificado de manera importante por efecto no sólo de la producción interna sino desde luego por las importaciones. En el año 2000 la disponibilidad de alimentos registraba ya 3000 calorías diarias por persona, duplicando las 1500 calorías de principios de la década de 1960.

\section{Autosuficiencia en granos}

Siguiendo la pauta de un país que se industrializa y crece de manera sostenida, la participación del sector agropecuario en el producto nacional va en descenso, alcanzando $10.1 \%$ en 2011, mientras que la industria y los servicios contribuyen con 45.3 y 44.6\%, respectivamente (CIA, 2013), al mismo tiempo que el peso demográfico del sector rural ya es ligeramente inferior al urbano, con 49.4\% en 2011, pero con una población económicamente activa muy alta $(34.8 \%)$ en proporción de la riqueza que genera el campo, superada apenas por el sector servicios en crecimiento $(35.7 \%)$ y muy por encima del sector industrial (29.5\%). Lo que pone en evidencia el desequilibrio existente entre sectores, con un claro sesgo urbano.

pondría en práctica una política de flexibilización del hukou para ayudar a la gente a registrarse como residentes urbanos en ciudades medianas y pequeñas, así como en pueblos, y asegurarles los beneficios de un ciudadano con registro urbano; aunque señaló que al mismo tiempo el gobierno continuaría ejerciendo un "control razonable" sobre la población de las ciudades más grandes, incluyendo Pekín y Shanghái (Bloomberg, 2013), pues en la actualidad existe ya un mercado donde se venden y compran los derechos inherentes al hukou en estas dos ciudades. 
Hay que señalar que el eje de la política agrícola en China es sin lugar a dudas mantener la autosuficiencia total en sus tres granos principales: arroz, trigo y maíz. Lo que debe cumplirse a pesar de cualquier obstáculo. Con 121.7 millones de hectáreas arables al terminar 2011, la superficie cultivable de China es escasa dado el volumen de su población y el dinamismo de la economía; restricción que cada día es mayor en función del aumento constante de los ingresos de sus habitantes, así como por los cambios en el patrón de consumo alimentario hacia uno cada vez más demandante. ${ }^{4}$ La tierra arable disponible actualmente es de 0.092 hectáreas por habitante, equivalente a $40 \%$ del promedio mundial. Se trata del tercer país del mundo por su superficie total, pero su frontera agrícola está agotada desde la época de Mao, al mismo tiempo que la mayor parte de los cultivos son de temporal. Su dotación de recursos, además, viene en retroceso, cuando el suelo y el agua son condición del mantenimiento de la seguridad alimentaria. ${ }^{5}$ Si ponemos como ejemplo el periodo de 1996 a 2004, la superficie agrícola disminuyó de 130 millones de hectáreas a 122 millones, al mismo tiempo que la población del país ha crecido en promedio 10 millones cada año y la demanda de bienes del campo va aparejada al alto crecimiento económico registrado en ese lapso.

Son múltiples los factores que han originado esta contracción del territorio productivo: el avance del desierto, ${ }^{6}$ la erosión de los suelos, ${ }^{7}$ fenómenos naturales recurrentes como las sequías y las inundaciones, la sobre explotación del suelo, así como por la política de reforestación y recreación de praderas, la contaminación ${ }^{8}$ y el agotamiento de acuíferos, ${ }^{9}$ la acelerada industrialización,

${ }^{4}$ Con el aumento de los ingresos no sólo se ha incrementado y diversificado la canasta de consumo, sino que los chinos han venido modificando sus hábitos alimentarios a favor del patrón occidental basado más en proteínas animales que en granos. Lo que significa una presión creciente sobre los recursos de la agricultura pues la ganadería exige hasta siete veces más recursos para la producción de una caloría de lo que se necesita para ser generada con producción vegetal.

${ }^{5}$ De las 23 provincias del país, 4 municipalidades, 5 regiones autónomas y 2 regiones administrativas especiales, 27 enfrentan el problema de la disminución de la tierra agrícola y 16 tienen superficie agrícola inferior a la cuota establecida por el Plan Nacional de Uso del Suelo (People's Daily Online, $10 / 24 / 2005)$.

${ }^{6}$ El fenómeno más impresionante por su velocidad es el avance cotidiano del desierto en el norte de China, probablemente el peor proceso puntual de desertificación en el mundo, con el sobrepastoreo de las provincias del norte y el occidente como uno de los factores más importantes (Deininger, 2008).

${ }^{7}$ En el noroeste de china la erosión del suelo se ha vuelto un problema especialmente importante que preocupa seriamente a los dirigentes del país por la seguridad en granos del futuro.

${ }^{8}$ Según el ministerio chino de agricultura, alrededor de 12.3 millones de hectáreas (más de $10 \%$ de la tierra arable del país) están contaminadas, al mismo tiempo que se estima que 37\% del territorio de China sufre de degradación del suelo (Watts, 2010/02/09).

${ }^{9} \mathrm{La}$ escasez de agua es un problema central en China, sólo cuenta con una disponibilidad de acuíferos menor a un tercio del promedio mundial. 
la urbanización y la construcción de infraestructura. En este marco, China ha adoptado el principio según el cual cualquier cambio del suelo agrícola para otros usos debería compensarse en otro punto del territorio, para lo cual se ha fijado una "línea roja" de 120 millones de hectáreas, más allá de la cual no se permitirán afectaciones de tierra arable. Pero ese límite ya se alcanzó y el cumplimiento de la norma no es lo estricto que debiera.

Todo lo anterior significa un enorme reto para la capacidad de China de mantener su actual autosuficiencia en granos, pues el país es ya importador neto de alimentos, aunque por supuesto no de sus tres granos estratégicos. Según estadísticas oficiales, en 2011 incrementó por octavo año consecutivo su producción de esas semillas, alcanzando 571 millones de toneladas (Lusardi y Odarda, 23/04/2012), cifra prevista como objetivo oficial para el año 2020 (National Bureau of Statistics of China, 2009). Lo que se logró con inversión masiva estatal y tecnologías de hibridación para elevar rendimientos en arroz a más de 10 toneladas por hectárea, e incrementarlos también en trigo y otros granos entre 1960 y $2004^{10}$ (Pandya-Lorch \& Spielman, 2009).

Es claro que a pesar de la liberalización de su economía, de las recomendaciones hechas por organismos internacionales y del predominio del pensamiento económico ortodoxo a nivel global, China sigue utilizando una absoluta intervención estatal para administrar el sector agropecuario y el comercio de los productos del campo; en particular, algunos precios internos como los de los granos ${ }^{11}$ están sujetos a controles con el fin de mantener la estabilidad de la oferta. Además, para lograr un alto grado de autosuficiencia, la adquisición de cereales está controlada en gran medida por el gobierno, siendo los principales distribuidores de granos las empresas estatales, como Sinograin (OMC, 2012a).

Igual que en todo el mundo occidental industrializado, el enfoque del gobierno chino sobre el sector agrícola se basa en el modelo de alta productividad, con tecnología convencional intensiva en capital, con insumos químicos, con mecanización y biotecnología, independientemente de sus externalidades sobre suelo, agua, aire, biodiversidad, productores y consumidores. Así, China produce $24 \%$ del total de granos del mundo, pero con $35 \%$ del consumo mundial de fertilizantes. Mientras que el incremento en el volumen del producto ha sido de 8 veces desde la década de 1960, el uso de fertilizantes

\footnotetext{
${ }^{10}$ El objetivo del sector científico es alcanzar 13.5 toneladas de arroz por hectárea en 2015 (Blinch, Lovalyova y Parsons, 2009).

${ }^{11}$ Los precios de garantía para granos se establecen cada año. Diseñados para ayudar a satisfacer la demanda en las provincias deficitarias, su aplicación está limitada geográficamente a 13 provincias excedentarias y que producen $80 \%$ de los granos comerciales del país y se otorgan varios meses después del periodo de cosecha.
} 
nitrogenados se multiplicó innecesariamente por 55 (Niu, 2010). En general, la modernización de la agricultura ha dependido de un uso importante de insumos dependientes de combustibles fósiles tales como petróleo y gas natural, que China importa en volúmenes que aumentan cada año, pero con menores impactos en productividad a lo largo del tiempo y crecientes efectos sobre el medio ambiente.

\section{Los apoyos a los productores}

Hoy, la base de la producción rural en China está integrada por 240 millones de hogares de agricultores con explotaciones de 0.65 hectáreas en promedio, que obedecen a una política de planificación central con metas cuantitativas de producción, principalmente para granos, y un sector de pastores nómadas en el noroeste que están dejando de serlo por efecto de una política expresa para asentarlos definitivamente.

Aproximadamente 642 millones de personas viven todavía en el campo y dependen significativamente de la agricultura para su sobrevivencia, por lo que la seguridad en la tenencia y la capacidad para invertir en la tierra son vitales, como también lo son otras fuentes de ingresos fuera de la agricultura como las empresas rurales y las remesas de sus familiares trabajando en otros puntos del territorio chino.

De ser el sector más castigado de la economía durante 2 mil años, al inicio del siglo xxi la agricultura dejó de ser gravada con el costo de los precios finales de los granos básicos que iban al sector urbano, así como con un sinnúmero de exacciones a nivel local que en su conjunto eran denominados "el fardo campesino". Hoy, por ley, el financiamiento público a la agricultura tiene que exceder el nivel de la tasa de expansión del gasto público total, aunque en un contexto de un rezago muy importante en el acceso de la población China a servicios básicos de educación, salud y seguridad social, en particular de los habitantes del campo y sobre todo de la cada vez más numerosa población de edad avanzada (OECD, 2011a).

El tránsito de gravar a los agricultores a apoyarlos es muy reciente, y desde 2004 comenzaron a recibir apoyo financiero, con precios de garantía mínimos para los principales granos, subsidios a los insumos y el inicio de la introducción de servicios de salud para elevar el nivel de vida rural. Ya en 2006 se eliminaron la mayoría de los impuestos agrícolas. Al mismo tiempo, las transferencias presupuestales han crecido constantemente desde fines de la década 
de 1990, subsidiando sobre todo la adquisición de agroquímicos, en particular fertilizantes, semillas mejoradas y maquinaria agrícola, así como cada vez más a través de pagos directos por unidad de superficie. Desde 2007 se pone en práctica el seguro agrícola como un nuevo apoyo y también se crean los pagos por cambio de uso del suelo, de agrícola a bosque, bajo el programa Green for grain. ${ }^{12}$ Fuera de la producción el financiamiento más importante es el del almacenamiento público de granos, seguido por diversos programas para apoyar la infraestructura agrícola, incluyendo instalaciones de irrigación y drenaje. Lo que la Organización para la Cooperación y el Desarrollo Económicos (OCDE) define como el indicador de apoyo al productor ${ }^{13}$ es nuevo en la historia de China, con 17\% del ingreso bruto agrícola en 2012, pero que todavía es inferior al promedio de $18.6 \%$ de los países de la OCDE, pero es más alto que en Sudáfrica (3\%), Brasil (5\%) o México (7\%) (OECD, 2013b). Hacia adelante, existen políticas que fijan precios techo a alimentos seleccionados, los subsidios al precio de los alimentos como tales son pequeños.

Como podemos ver, se trata de un modelo con características que los economistas ortodoxos descalificarían por ineficiente, pues no se basa en grandes extensiones de tierra ni en propietarios privados especializados con fines de ganancia, ni en sumas masivas de capital privado. Y sin embargo, se cumple con el objetivo de la autosuficiencia de manera reiterada a lo largo de tres décadas, lo que no es esperado por el modelo ortodoxo, si no es con plena apertura del sector. Hay que subrayar que ningún país industrializado tiene una agricultura de corte liberal en el sector de básicos y productos estratégicos del campo, sino que al igual que China las economías más ricas han desarrollado un sector agropecuario que cumple con objetivos de autosuficiencia fomentando una canasta de productos considerados estratégicos antes que rentables. Esta política le cuesta al erario, al consumidor y al contribuyente, y donde de manera universal y en primer lugar están los granos, luego, y según los recursos naturales y financieros de cada economía, otros alimentos de origen animal o vegetal, hasta llegar a incluir fibras o adoptar el enfoque de regiones, de grupos de productores vulnerables o de especies animales y vegetales sensibles. Los productos

\footnotetext{
${ }^{12}$ En 1999 se puso en marcha el programa piloto Green for Grain con los objetivos de alentar la forestación, revertir el proceso de degradación ecológica y erosión del suelo y reducir la explotación excesiva de las tierras sensibles. Para finales de 2008, habían pasado a ser bosques 8.2 millones de hectáreas de tierras cultivables y 27 millones de unidades familiares rurales participaban en ese programa de pagos (OMC, 2012a).

${ }^{13} \mathrm{El}$ indicador de apoyo al productor (PSE por sus siglas en inglés) incluye apoyo en servicios generales a la investigación agrícola, infraestructura, inspección, comercialización y promoción, así como subsidios a los consumidores (OECD, 2013).
} 
seleccionados reciben transferencias de fondos para garantizar la oferta, fortalecer la productividad, los ingresos de los productores, el desarrollo regional, el cuidado y la remediación ambiental, teniendo en el centro la idea de eliminar vulnerabilidad y garantizar el funcionamiento en los sectores definidos como medulares para el país. Todo con el fin de mantener una estructura macroeconómica interconectada y funcionando adecuadamente. Si esto fuera dejado al libre mercado no se cumplirían los objetivos señalados, pues las razones que los sustentan no son solamente económicas y ningún mercado reconoce variables políticas ni sociales que hay que contemplar cuando de alimentos se trata, pues la tecnología no ha logrado generar sustitutos perfectos de lo que comemos. Y eso, los chinos lo tienen muy claro.

Sin embargo, es notorio que sin los recursos de un país desarrollado, China ha obtenido los mismos resultados que las potencias capitalistas en el terreno de la autosuficiencia. Pero debemos detenernos para analizar algunos aspectos. En primer lugar, recordemos que a medida que la agricultura se industrializaba, sobre todo en el periodo de la segunda posguerra con la tecnología de la revolución verde, las economías capitalistas más desarrolladas tuvieron que generar instrumentos para arraigar a los agricultores en el campo y por décadas han tenido que administrar la emigración de fuerza de trabajo del campo a la ciudad con diversos programas de transferencias y complementación de ingresos extra sectoriales para la estabilización y la elevación del ingreso total promedio rural hasta el nivel de los ingresos urbanos promedio.

A diferencia de Occidente y en ausencia de libre circulación de la fuerza de trabajo en el territorio, el régimen político administrativo de China ha permitido introducir la lógica mercantil sólo hasta el punto en que le resulta práctico para los fines de política agropecuaria y macroeconómica, sin liberalizar la economía en los términos señalados por el Banco Mundial y la Organización Mundial de Comercio. El gobierno central no necesita seducir a los agricultores con instrumentos económicos atractivos para inducirlos a no emigrar y cumplir con sus objetivos de política económica, simplemente no es legal abandonar el campo para trabajar en la ciudad sin autorización, no importa cuán pobres sean. No tienen más opción que trabajar en el campo y cumplir con las metas señaladas por el gobierno central.

Esto ha hecho que su política agrícola sea más barata que la de los países industrializados, por el simple hecho de no tener que invertir en lograr la convergencia de ingresos entre el campo y la ciudad, sino que ha podido dejar rezagados a los campesinos que hasta ahora son el sector más pobre del país; razón por la cual hay un flujo persistente de emigrantes a las zonas industriales y urbanas, con permiso o sin él. 
Lo que hace prever que, junto con la política de urbanización del país, para el año 2030 cuatrocientos millones de habitantes se hayan desplazado a las ciudades, a pesar del intento de mejorar las áreas rurales bajo el concepto reciente de "nuevo campo socialista"; habiendo dejado ya como secuela una composición demográfica dominada por ancianos, mujeres y niños que anuncia una incipiente escasez de mano de obra y un concomitante aumento del costo del trabajo y abriendo la puerta a la necesidad de modernización del sector en el sentido de menos intensivo en trabajo y más en maquinaria y capital.

En última instancia, el objetivo principal de la política en el campo es salvaguardar la seguridad alimentaria mediante el aumento de la capacidad de producción y la mejora de la competitividad. ${ }^{14}$ En el Duodécimo Plan Quinquenal (2011-2015) se reafirman los compromisos que figuran en planes anteriores fortaleciendo el apoyo a los agricultores, dándoles más beneficios y promoviendo la modernización, con el fin de mejorar sus niveles de vida y dinamizar el mercado interno. También se hace hincapié en la diversificación de la economía rural mediante el desarrollo del turismo, los combustibles alternativos, las inversiones en infraestructura, una mejor urbanización de pueblos y aldeas y el mejoramiento de los servicios públicos con inclusión de la educación y la capacitación.

\section{Los ingresos}

La brecha de los ingresos de los campesinos frente a los ingresos urbanos medios en China es tan importante que llevaría a los agricultores, naturalmente y de manera masiva, a abandonar el campo buscando un ingreso mejor en otro sector, pero han sido contenidos por la política que los fija en su lugar de nacimiento. Las autoridades chinas han planteado ya la necesidad de poner en el centro de su política de crecimiento económico la igualación de las condiciones de vida del sector rural con el urbano, pues de otra manera se rezagaría el crecimiento del mercado interno. De 2004 a 2011 los documentos anuales de máxima prioridad del gobierno, denominados siempre Documento No.1, ponen como tema central al campo y a los campesinos junto con el objetivo de "incrementar los ingresos de los agricultores" a través de ajustar la estructura agrícola, incrementar los empleos rurales, aumentar la inversión rural, profundizar la reforma del campo y acelerar el trabajo de la ciencia y la tecnología agrícolas. "Construir un campo socialista" fue la clave prioritaria

\footnotetext{
${ }^{14}$ En el plan se establecen objetivos más específicos, que incluyen un aumento de 50 millones de toneladas en la capacidad de producción de granos, definidos como arroz, trigo, maíz y frijol de soya.
} 
del plan quinquenal 2006-2010 y la economía rural también está en el centro del plan quinquenal 2011-2015, el décimo segundo de China, y que pretende reequilibrar el crecimiento que ha estado dirigido contundentemente por exportaciones e inversión ancladas en el sector urbano. Mejorar el bienestar en el campo e incrementar el ingreso rural son concebidos como críticos para hacer crecer la demanda doméstica, como tarea mayor para transformar el patrón de crecimiento económico de China ${ }^{15}$ (OECD, 2011a).

Según el Bureau of Statistics del gobierno chino el ingreso rural per cápita neto en 2012 fue de 7917 yuanes, tras un aumento de 10.7\%; menos de un tercio del ingreso urbano por habitante de 24565 yuanes y que tuvo un incremento real de 9.6\%. La misma fuente señaló que el coeficiente de Gini fue de 0.474 , ligeramente superior a los niveles de desigualdad en el ingreso de Estados Unidos, la primera potencia mundial ${ }^{16}$ (Bloomberg, 2013). Lo que nos recuerda que habiendo sido la economía más igualitaria del mundo antes de la apertura económica, China es hoy una economía cada vez más polarizada, con 600 millones de pobres todavía, mayoritariamente en el campo.

\section{Comercio agrícola}

Dada la restricción absoluta de recursos para la agricultura, así como en relación con su dinámica de crecimiento, es claro que China se volvió un importador neto de alimentos, comprando en el mercado mundial bienes intensivos en suelo (soya, algodón, cebada, hule y aceites de soya y palma) y exportando bienes intensivos en fuerza de trabajo (pescado, frutas, hortalizas y bienes agrícolas procesados).

El gobierno chino ejerce control sobre el comercio de granos y otros bienes estratégicos a través de empresas propiedad del Estado, cuotas y licencias de importación y exportación, impuestos a la exportación, reducciones temporales de aranceles, medidas sanitarias y fitosanitarias, impuestos y subsidios (USDA, 2012). La protección de la agricultura en frontera hace que los productos del campo tengan un arancel promedio de $15.1 \%$, superior a $8.6 \%$ de todos los demás bienes. Los aranceles más elevados, que pueden alcanzar hasta $65 \%$,

${ }^{15}$ El primer documento de política emitido por el comité central del PCC y el Consejo de Estado, es el Documento Central No.1.Y 2010 es el séptimo año consecutivo en que el documento se centra en los problemas rurales (Chinese Government, 2010). El plan quinquenal 2011-2015 lo incluye como un tema prioritario.

${ }^{16}$ Hay otros cálculos que señalan que la medida real de la desigualdad en China es superior a la que reconoce su gobierno, pues no se incluyen en la contabilidad las fuentes ilegales de enriquecimiento que son considerables (Buckley, 2013). 
se aplican a los cereales. Arroz, trigo, azúcar, algodón y algunos fertilizantes químicos están sujetos a cuotas arancelarias, así como a ser comercializados por empresas del Estado, mientras que las exportaciones de arroz, maíz, algodón y tabaco están altamente controladas, sólo se realizan mediante empresas estatales y están sujetas a cuotas de exportación (OMC, 2012a).

El comercio agrícola en 2012, al igual que en años anteriores, apenas representó $3 \%$ de sus exportaciones y $6,6 \%$ de sus importaciones totales, con un ritmo de crecimiento de las importaciones muy relevante; lo que genera un déficit comercial creciente en este rubro, en contraste con la balanza comercial global que es superavitaria por 231 mil millones de dólares, 50\% superior a 2011.

Con importaciones en 2010 de 67 mil millones de dólares estadounidenses y exportaciones de sólo 36 millones de dólares, en 2012 el déficit comercial agrícola de China aumentó 34\% en comparación con 2011, llegando a 57 530 millones, como resultado de importaciones por 120078 millones de dólares y exportaciones por 62550 millones, 16 y 3.5\% superiores al año anterior, respectivamente. Esto fue resultado tanto del incremento en el volumen comercializado como del aumento en el precio de las materias primas. Los productos que registraron las más altas tasas de crecimiento en las importaciones fueron arroz (290\%), trigo (237\%), maíz (197\%), carne bovina (187\%), semillas de colza (134\%), aceite de colza (107\%), alfalfa (60\%), aceite de soya (60\%), algodón (53\%), azúcar (34\%), maderas en bruto (34\%), aceite de oliva (29\%) y leche en polvo (27\%). Principalmente materias primas.

De mucha relevancia por su peso absoluto fueron las importaciones de frijol de soya ${ }^{17}$ (29\% del total agrícola importado), algodón (10\%) y aceite de palma (5\%), donde Estados Unidos y Brasil son los más importantes proveedores. Sus importaciones están concentradas en 90\% en Estados Unidos (25\%), Brasil (16\%), la Unión Europea (UE) (7\%) y 13 países más.

Las exportaciones agrícolas chinas están también muy concentradas en 19 países y la UE. En 2012 estos destinos concentraron el 88,8\% de las ventas agrícolas al extranjero, con la participación predominante de Japón (19\%), UE (12\%), eu (11\%), Hong Kong (9\%) y Corea del Sur (7\%).

Los principales productos de exportación fueron ajos frescos o refrigerados, productos de la pesca congelados o en conserva (filetes de pescado, mariscos, anguilas); manzanas (frescas y jugo); alimentos para mascotas; aditivos de alimentos para animales y mandarinas (Odarda y Viola, 2013).

\footnotetext{
${ }^{17}$ El crecimiento porcentual no es de los mayores porque desde mediados de la década de 1990 China se convirtió en uno de los mayores demandantes de soya en el mundo.
} 
Estos datos nos permiten constatar que la composición de la balanza comercial agropecuaria china le es totalmente adversa, no sólo por el amplio déficit, sino porque el contenido también es cualitativamente asimétrico. China importa alimentos que no tienen sustitutos y exporta una gama de productos que no son relevantes para la economía de sus socios comerciales.

\section{Inocuidad}

Junto a su creciente déficit en la balanza agroalimentaria, China no ha traspuesto el umbral de una política basada en generar el volumen de alimentos que necesita su economía, hacia un modelo centrado en la calidad y la inocuidad de sus productos. En ese sentido todavía es una nación subdesarrollada.

Nos hemos familiarizado a través de los medios de comunicación con temas relativos a la falta de control gubernamental sobre la calidad de los alimentos que entran al mercado en China: melamina en la fórmula láctea para bebés, coles rociadas con formaldehido, millones de cápsulas medicinales conteniendo cromo, aceite comestible para reciclar extraído del drenaje, chícharos artificiales pintados de verde. (McDonald, 2012), son ejemplos que dan muestra de que la normatividad existente y su cumplimiento son insuficientes.

Pero no sólo la existencia de quienes tratan de obtener ganancias de manera ilícita adulterando alimentos es inaceptable, sino que el patrón tecnológico dominante en la producción en campo está integrado por un monto importante de tóxicos por basarse en la utilización excesiva de químicos, al mismo tiempo que existen condiciones propicias para la diseminación de enfermedades como la gripe porcina o la fiebre aviar que no son atendidos a satisfacción por las autoridades desde su fase preventiva. No es sólo una cuestión de fortalecer la ley y su cumplimiento, sino de una concepción sobre lo que es o no sano para el consumidor, el productor y el medio ambiente. Y no es el enfoque que se pone en práctica, se protege la producción de alimentos sólo en su capacidad productiva, pero no en la calidad.

\section{Un campo moderno empobrecido}

Junto a este escenario existe un tema relevante que vale la pena mencionar aunque no profundicemos en él en estas páginas, se refiere a la homogeneización del campo en China como resultado de una política que ha destruido las prácticas y conocimientos autóctonos de producción con un enfoque produc- 
tivista, una tecnología y un paquete tecnológico de aplicación universal que presionan y agotan la capacidad de recuperación de la naturaleza; igual que ha sucedido en todos los países donde la producción se ha regido por la tecnología de la Revolución Verde.

Es en las provincias occidentales y las regiones autónomas (Tibet, Sichuan, Qinghai, Mongolia Interior y Xinjiang), las que están más alejadas de las zonas dinámicas de la costa, en donde más se conservan las producciones primarias y las prácticas tradicionales, justamente por su acceso difícil. Pero, con antecedentes desde la fundación de la República Popular, desde 1985 las autoridades centrales chinas han puesto en práctica una serie de políticas con el objetivo de integrar a estas zonas de manera tajante y radical al modelo económico puesto en marcha desde la apertura, a través de implantar la lógica de producción agrícola de alto rendimiento y con el objetivo de modernizar la cría de animales para volverla completamente mercantil, con los argumentos de elevar los niveles de vida de los habitantes autóctonos, así como de combatir la degradación de praderas y prevenir desastres naturales en las partes bajas de China.

En este contexto se impulsa el establecimiento de pastores en áreas fijas a costa del abandono de su vida trashumante y de su estilo de vida, así como a costa de la transformación radical de sus culturas, de su sinificación, con políticas de reubicación de pastores nómadas y de otras formas de residentes rurales, tales como: proveer de alojamiento con acceso a electricidad, agua, transporte, escuela y servicios de salud, así como modernizar y "hacer entrar en el desarrollo" a las minorías étnicas consideradas económicamente "atrasadas". En los hechos se trata de una violenta integración de la población que ofrece algún tipo de resistencia a las políticas centrales por razones históricas, culturales y también políticas, desde la meseta tibetana con décadas de movimientos independistas hasta la población musulmana de uigures donde también se registran brotes de insurrección. Para el gobierno central, la integración implica el fomento de la migración de la etnia mayoritaria en el territorio - han — a las regiones mencionadas, hasta volverse población mayoritaria, que la escuela y la alfabetización vuelvan el mandarín la lengua dominante ${ }^{18}$, que sus diferencias culturales sean puestas al servicio del sector turístico con las adaptaciones que

\footnotetext{
${ }_{18}$ Cuatrocientos millones de chinos no hablan mandarín, a pesar de décadas en que el PCC ha promovido esa lengua como medio de integración nacional, por sobre la existencia de miles de dialectos y lenguas de las minorías. Pero esa campaña ha provocado resistencia que ha llegado a la violencia en algunos casos, así como preocupación por la dimensión del territorio nacional y la falta de inversión en educación, especialmente en las áreas rurales pobres. El vocero del Ministerio de Educación, Xu Mei, dijo que sólo 70\% de la nación hablaba mandarían, incluyendo a aquéllos que no lo hablan bien (Reuters, 5/09/13).
} 
ello implique para su forma de vida, entre otros aspectos. La homogeneidad es el precio que el progreso del capitalismo y el control político demandan en el territorio chino.

\section{Más allá de sus fronteras}

Recordemos que el éxito económico en China se ilustra con que la economía creció 20 veces entre 1978 y 2008, a una tasa promedio anual de 9.8\%. El ingreso per cápita creció 50 veces y alrededor de 500 millones de personas dejaron de ser pobres con un proceso de industrialización que las economías de la mayoría de los países industrializados tardaron cien años o más en completar, mientras que en el caso chino se trató de sólo tres décadas. Sin embargo, China no ha alcanzado el rango de nación "desarrollada" en los términos convencionales, pero ha excedido ya lo que su territorio ofrece para la agricultura y demanda de manera indefinida más recursos de fuera a través del comercio y de cualquier otro medio posible, como condición lógica del "buen desempeño económico" implicado en el mantra del crecimiento económico infinito con un alto costo sobre la naturaleza y sobre las poblaciones ligadas a esos recursos, dentro y fuera de sus fronteras (Trápaga, 2011).

En el caso de la agricultura, la creciente restricción de recursos que impide ya el cumplimiento del objetivo de autosuficiencia a cien por ciento o más allá, ha llevado al gobierno chino a salir de sus fronteras con la política de "going out" o "going global" buscando ampliar sus fronteras agrícola, energética, minera, hídrica y relativa a recursos naturales, a través de comprar, alquilar, obteniendo por concesión y cualquier otro medio posible los recursos que le son necesarios para mantener su crecimiento económico y su posición como potencia global. China se incorpora a la tendencia mundial de ocupación de tierras y aguas en distintos países, preferentemente subdesarrollados, adquiriendo tierra agrícola en su periferia asiática, en África, en América Latina, en Australia, en el marco de un proceso mundial de acaparamiento de tierras, reconocido incluso por Naciones Unidas y por el Banco Mundial.

China cuenta con inversiones en procesos agrícolas de producción fuera de sus fronteras que le permiten tener mayor control sobre el modelo de agricultura que ha elegido, reducir su exposición frente a los mercados internacionales y al mismo tiempo tener bajo su dominio los recursos que no tiene en su territorio pero que necesita para la producción, buscando garantizar alternativas para el abasto alimentario de largo plazo, ${ }^{19}$ al igual que lo hacen otras

\footnotetext{
${ }^{19}$ China busca incrementar su producción de arroz fuera de sus fronteras, principalmente en las regiones asiática y africana.
} 
economías con liquidez, pero deficitarias en agua y suelos para la agricultura, entre los cuales sobresalen Qatar, los Emiratos Árabes, Japón y Corea del Sur, que compran o toman en alquiler grandes extensiones de tierra, ya sea a través de sus gobiernos o por privados, con el fin de producir directamente alimentos vegetales y animales, plantaciones forestales, cultivos para exportación, agrocombustibles y hasta para especulación (Trápaga, 2012).

Es necesario señalar que como todas las economías que se modernizan la adopción del patrón alimentario occidental basado en proteínas de origen animal es totalmente ineficiente si pretende competir con un patrón centrado en vegetales, pues se necesitan muchísimos más recursos para satisfacer el mismo nivel de necesidades calóricas con el sistema vigente en los países ricos. Lo que en presencia de una creciente demanda por efecto de ingresos al alza de la población, estimula la búsqueda de cada vez más recursos naturales dentro o fuera de los límites territoriales de un país. En este contexto, en junio de 2008 China adquirió 101171 hectáreas en Zimbabue, en el mismo año invirtió 800 millones de dólares en Mozambique para modernizar la agricultura con el fin de exportar de arroz a China continental, también adquirió 2.8 millones de hectáreas en la República Democrática del Congo para crear la plantación más grande del mundo de palma para obtener aceite, un insumo de bajo costo para la industria alimentaria y del que es altamente deficitario. En 2009 adquiere en Tanzania y Zambia un total de 4.8 millones de hectáreas para producir arroz y biocombustibles. A lo anterior podríamos agregar el caso de las cuantiosas inversiones de China en Argentina y Brasil para la producción de soya y granos, en la pampa húmeda y el Cerrado, respectivamente.

Si nos referimos a los bosques, por ejemplo, el Banco Mundial calcula que la expansión de China en busca de madera y celulosa la orienta hacia los trópicos, dada la alta productividad natural forestal de esas latitudes, afectando también en 2008 una superficie de 3.4 millones de hectáreas de bosques naturales, mientras que las áreas forestales de su país gozan de toda la protección (Deininger, 2008).

Resulta lógico pensar que esta tendencia se agravará, pues además de su creciente déficit de recursos primarios y de sus malas prácticas agropecuarias en términos ecológicos, China está viendo mutar sus condiciones naturales por el cambio climático, pero no a su favor, pues se estima que el calentamiento global puede hacer caer la productividad agrícola en su territorio entre $5 \mathrm{y}$ $10 \%$ hacia 2030, a pesar de acciones de mitigación, con efectos principalmente sobre trigo, arroz y maíz. 
El sistema agrícola establecido por el poder central ha logrado impresionantes resultados en los últimos 30 años, pero las políticas hacia el sector deben incorporar de manera urgente el cuidado de los recursos naturales, de los productores y de los consumidores con una planificación en una dirección distinta a la vigente durante décadas, pues el modelo se desarrolla en un callejón sin salida.

Para el gobierno chino la modernización de la agricultura implica volverla comercial, dado que cuenta con un acervo creciente de tierras en el extranjero para complementar la oferta de bienes básicos que le permitirá liberar suelos domésticos para cultivos de alta rentabilidad para sectores de altos ingresos o para la exportación. Sólo así será posible lograr una creciente diversificación, así como fomentar segmentos de muy distintos tipos y sofisticación, desde para producir más bienes de la canasta básica (soya y algodón, por ejemplo), hasta para generar agrocombustibles o especializar ciertas zonas en productos orgánicos.

Esto, seguramente amenazaría la viabilidad de largo plazo de las aldeas y pueblos de agricultores familiares, pues uno de los supuestos de la modernización es que las unidades de producción crezcan hasta poder tener economías de escala, pero eliminando productores que tendrán que ser ubicados en el sector urbano, reproduciendo con ello el modelo polarizado del capitalismo moderno y liquidando las culturas rurales de uno de los países de mayor diversidad biológica del planeta.

\section{Conclusiones}

Si nos atenemos a la mera cronología, el siglo veinte termina con el derrumbe del sistema socialista de corte soviético y la incorporación al capitalismo de casi todos los países que pertenecían al bloque; paralelamente se registra un proceso vertiginoso de ascenso económico en China, sólo tres décadas después de su fundación; un esquema de acumulación voraz, un capitalismo híbrido con un mercado de dinamismo excepcional y un sector de planificación central que el gobierno de ese país designa como "socialismo de mercado" o "socialismo con características chinas" y donde la agricultura ha combinado el modelo probado como exitoso de las potencias capitalistas con el mejor ejemplo de movilidad legalmente regulada de la fuerza de trabajo y otros instrumentos de la planificación central, como la fijación de cuotas de producción o la comercialización por empresas de Estado, pero con costos muy altos en términos sociales y ambientales. 
El ejemplo chino derrumba una vez más el postulado ortodoxo de que sólo con unidades de producción de gran escala se puede tener alta productividad, pues en su territorio ha sucedido exactamente lo contrario, con explotaciones de muy pequeña escala y una base de productores familiares ha logrado un objetivo que otros países con infinitamente más recursos, como México, han visto imposible de alcanzar.

Ya no es China, sino el planeta, el que deberá pagar esta nueva cuenta por la exigencia del modelo global de producción con aspiraciones de crecimiento ilimitado en un mundo finito, igual que históricamente ha sucedido y sigue pasando para sostener la dinámica de las potencias del mundo y de las economías emergentes. China es una potencia y no sería lógico que renunciara a serlo en la dinámica de un planeta regido por la competencia y la ganancia; pero los recursos ya no bastan para mantener la demanda desmedida de las economías más industrializadas, no sólo para la producción, sino como sumideros de desechos.

Alguien más pagará la cuenta, como siempre. En primera instancia son las economías más débiles y los habitantes pobres del mundo; es la humanidad entera y el planeta azul.

\section{Bibliografía}

Blinch, Russell, Svetlana Kovalyova \& Claudia Parsons, RPT-SPECIAL RePORT-

The fight over the future of food, Reuters, http://www.reuters.com/article/2009/11/10/food-idUSN09174220091110

Bloomberg, China Rural-Income Gains Aid Shift Toward Consumption, Bloomberg NEWs, Jan 29, 2013 http://www.bloomberg.com/news/2013-01-29/china-rural-income-gains-aid-shift-toward-consumption.html

Buckley, Chris, China Issues Proposal to Narrow Income Gap, The New York Times, February 5, 2013. http://www.nytimes.com/2013/02/06/world/asia/chinaissues-plan-to-narrow-income-gap.html?_r=0

Central Intelligence Agency, CIA World Factbook 2013, https://www.cia.gov/library/publications/the-world-factbook/geos/ch.html

Chinese Government (2006a), Development, China Fact File, Chinese Government's Official Web Portal, http://english.gov.cn/2006-02/08/content_182638.htm

-_ (2005), History, China Fact File, Chinese government's Official Web Portal, History, http://english.gov.cn/2005-08/06/content_24233.htm

(2006b), Township enterprises, China Fact File, http://english.gov.cn/200602/08/content_182632.htm 


\footnotetext{
02/01/content_1525464.htm
}

- Consecutive No. 1 central documents target rural issues, Monday, Feb-

Deininger, K. (Land Tenure Adviser) (2008), Large scale land acquisition: what is happening and what can we do?, World Bank team (ARD, DEC, LEG, SDV), 2010-01-24_Deiniger_Large-Scale-land-acquisition(1).pdf http://www.donorplatform.org/load/9812

Lusardi, Matías y Omar Odarda (consejero agrícola), Agrichina 017, Beijing, 23 de abril de 2012, Consejería Agrícola, Embajada de Argentina en la República Popular China, Ministerio de agricultura, ganadería y pesca. Presidencia de la nación, http://www.agrichina.org/download/17-Newsletter-Agrichinaabril-2012.pdf

McDonald, Mark, From Milk to Peas, a Chinese Food-Safety Mess, International Herald Tribune, The Global Edition of The New York Times, IHT Rendevouz, June 21, 2012, http://rendezvous.blogs.nytimes.com/2012/06/21/from-milkto-peas-a-chinese-food-safety-mess/

National Bureau of Statistics of China, China Energy Statistical Yearbook 2009, Beijing, China Statistics Press, 2009.

Niu Shuping (reporter) and Beijing Newsroom, China needs to cut use of chemical fertilizers: research, REUTERS, Editing by Chris Lewis and Jerry Norton), January 14, 2010, http://www.reuters.com/article/2010/01/14/us-china-agriculturefertiliser-idUSTRE60D20T20100114

$\operatorname{OECD}$ (2011a), China-AgriculturalPolicyMonitoringandEvaluation2011,http://www. oecd.org/documentprint/0,3455,en_2649_33797_48713462_1_1_1_1,00.html

- (2011b), China, in Agricultural Policy Monitoring and Evaluation 2011: OECD Countries and Emerging Economies, OECD Publishing. http://dc.doi. org/10.1787/agr_pol-2011-23-en http://www.keepeek.com/Digital-AssetManagement/oecd/agriculture-and-food/agricultural-policy-monitoring-andevaluation-2011/china_agr_pol-2011-23-en

__ (2013), Food security, the People's Republic of China avoiding the middleincome trap: policies for sustained and inclusive growth, September 2013, p. 12, http://www.oecd.org/china/China-Brochure-eng-September2013.pdf

Odarda, Omar (Consejero agrícola) y Lic. Hernán Viola, Informe. Análisis del comercio agrícola chino en 2012, Consejería Agrícola, Embajada de Argentina en la República Popular China, DOC/CAP/011-2013, Beijing, 31 de mayo de 2013, Ministerio de agricultura, ganadería y pesca. Presidencia de la nación, http:// www.aiera.org/newsletter/link/InformeComercioAgricolaChino\%202012.pdf OMC (2012a), China, WT/TPR/S/264/Rev.1, Página 113, IV: Políticas comerciales, 
Economía Informa núm. 384 enero - febrero • 2014

por sectores, Agricultura, http://www.agrichina.org/admin/kindeditor-4.1.2/ attached/file/20121024/20121024162008_0000.pdf

___ (2012b), Examen de las Políticas Comerciales. Resumen, WT/TPR/S/264 Página viii, http://www.wto.org/spanish/tratop_s/tpr_s/s264_sum_s.pdf

Pandya-Lorch, Rajul \& Spielman, David J., Millions Fed: Proven Successes in Agricultural Development, International Food Policy Research Institute, November 2009, 26 pp. http://www.ifpri.org/book-5826/ourwork/programs/2020vision-food-agriculture-and-environment/millions-fed-intiative

People's Daily Online, China's arable land decreases to 102.4 million hectares, October 24, 2005, http://english.peopledaily.com.cn/200510/24/eng20051024_216474. html

Reuters, China: 400 Million Cannot Speak Mandarin, The New York Times, September 5, 2013, http://www.nytimes.com/2013/09/06/world/asia/china400-million-cannot-speak-mandarin.html?_r $=0$

Trápaga Delfín, Yolanda, El fin de la frontera agrícola y el acaparamiento de tierras en el mundo, Investigación Económica, Facultad de Economía, UNAM, 2012.

____ "El medio ambiente o los límites del buen desempeño económico de China" en: Jorge Eduardo Navarrete (coord.), La huella global de China. Interacciones internacionales de una potencia mundial, CEICH, UnAm, Colección Prospectiva Global, Primera edición, 2011, 534 pp., p 491-534.

United Nations, General Assembly, 20 January 2012, Human Rights council, Nineteenth session, Agenda item 3, Promotion and protection of all human rights, civil, political, economic, social and cultural rights, including the right to development, Report of the Special Rapporteur on the right to food on his mission to China (15-23 December 2010), http://www.srfood.org/images/stories/pdf/ officialreports/20120306_china_en.pdf

USDA, Economic Research Service. China, last updated May 30, 2012, http://www. ers.usda.gov/topics/international-markets-trade/countries-regions/china/ trade.aspx\#.UjYWd9IyKXs

Watts, Jonathan (Asia environment correspondent), Chinese farms cause more pollution than factories, says official survey, The Guardian, Tuesday 9 February 2010, http:// www.theguardian.com/environment/2010/feb/09/china-farms-pollution 